\title{
Acute ingestion of resistant starch reduces food intake in healthy adults
}

\author{
Caroline L. Bodinham ${ }^{1}$, Gary S. Frost ${ }^{2}$ and M. Denise Robertson ${ }^{1 *}$ \\ ${ }^{1}$ Diabetes and Endocrinology, Postgraduate Medical School, University of Surrey, Guildford, Surrey GU2 7WG, UK \\ ${ }^{2}$ Imperial College London, London W12 ONN, UK
}

(Received 23 March 2009 - Revised 17 September 2009 - Accepted 21 September 2009 - First published online 27 October 2009)

Resistant starch (RS), a non-viscous dietary fibre, may have postprandial effects on appetite regulation and metabolism, although the exact effects and mechanisms are unknown. An acute randomised, single-blind crossover study, aimed to determine the effects of consumption of $48 \mathrm{~g}$ RS on appetite compared to energy and available carbohydrate-matched placebo. Twenty young healthy adult males consumed either $48 \mathrm{~g}$ RS or the placebo divided equally between two mixed meals on two separate occasions. Effects on appetite were assessed, using an ad libitum test meal and 24-h diet diaries for energy intake, and using visual analogue scales for subjective measures. Changes to postprandial glucose, insulin and $\mathrm{C}$-peptide were also assessed. There was a significantly lower energy intake following the RS supplement compared to the placebo supplement at both the ad libitum test meal (5241 (SEM 313) v. 5606 (SEM 345) kJ, $P=0.033)$ and over the $24 \mathrm{~h}(12603$ (SEM 519) v. 13949 (SEM 755$) \mathrm{kJ}$ $P=0.044)$. However, there was no associated effect on subjective appetite measures. Postprandial plasma glucose concentrations were not significantly different between supplements, but there was a significantly lower postprandial insulin response following the RS supplement $(P=0 \cdot 029)$. The corresponding C-peptide concentrations were not significantly different, although the ratio of C-peptide to insulin was higher following the RS supplement compared to placebo $(P=0.059)$. These results suggest that consumption of $48 \mathrm{~g}$ RS, over a 24 -h period, may be useful in the management of the metabolic syndrome and appetite. Further studies are required to determine the exact mechanisms.

\section{Fibre: Appetite: Postprandial insulin}

The incidence of obesity has increased over recent years and has reached an epidemic extent ${ }^{(1)}$; this is also associated with other health issues such as an increased prevalence of insulin resistance and type 2 diabetes. Various strategies have been investigated to determine how this problem could be tackled and to prevent a further increase in body weight and the associated conditions. These strategies include investigations into individuals' diets and specific dietary components that could be altered to subsequently impact on weight, insulin resistance and type 2 diabetes. One such component is dietary fibre. This diverse group of carbohydrates has been proposed to increase satiety ${ }^{(2)}$ and therefore may have a beneficial role in weight management. Dietary fibre may increase satiety by several mechanisms and different fibres may exert their effects by different means although the specific processes are not known. Proposed mechanisms include the lower energy density of fibre foods, the impact of fibre on delaying gastric emptying, which subsequently could prolong the feeling of fullness, and fibre slowing glucose absorption, which may prolong satiety ${ }^{(3)}$. There is also evidence to suggest that dietary fibres may influence insulin sensitivity ${ }^{(4)}$ independently to any effects on appetite.

Resistant starch (RS), a fermentable carbohydrate, has been proposed to have properties similar to dietary fibre ${ }^{(5)}$ and therefore could also affect satiety and exert a beneficial role in weight regulation. However, as RS is a non-viscous fibre and is not thought to affect gastric emptying or the absorption of nutrients ${ }^{(6)}$, the effects on satiety, if indeed there are any, are unclear. Use of RS may be a more advantageous means of increasing fibre intake compared to traditional fibres, as it is easy to incorporate into everyday foods ${ }^{(7)}$ without adverse alterations to either taste or texture ${ }^{(8)}$.

There are few studies that have investigated the effects of including RS into the diet and the consequent effects on appetite. Animal studies show consistent positive effects on appetite regulation and insulin sensitivity; however, data in human studies are mixed ${ }^{(9)}$. In particular, there are no current studies in human subjects that have explored the effects on appetite of including RS in mixed meals when compared to energy and carbohydrate-matched placebo. Of the four classifications of RS, the majority of studies looking at effects on appetite have used type 2 , which are intrinsically resistant, and type 3 , which are retrograded starches.

The postprandial effects of consumption of RS are also not known; however, as a non-viscous fibre, it would not be predicted to affect glucose absorption. Nevertheless, it has been shown in a recent study that short-term consumption of RS type 2 improves postprandial glucose metabolism in healthy individuals ${ }^{(6)}$.

The present study therefore aimed to investigate the acute effects of including $48 \mathrm{~g}$ of RS type 2 on energy intake, subjective measures of appetite and changes to postprandial

Abbreviations: RDS, rapidly digestible starch; RS, resistant starch.

*Corresponding author: Margaret Denise Robertson, fax +44 1483 688501, email m.robertson@surrey.ac.uk 
glucose and insulin, compared to a placebo supplement in a randomised, single-blind balanced crossover, in which the $48 \mathrm{~g}$ were divided equally between breakfast and lunch (providing $24 \mathrm{~g}$ at each meal) and consumed as part of these mixed meals.

\section{Experimental methods}

\section{Subjects}

Twenty young, healthy, adult males, aged 19-31 years, with a mean BMI $23.2($ SEM 0.65$) \mathrm{kg} / \mathrm{m}^{2}$ (Table 1) participated in the study. Subjects had no history of gastrointestinal disease or endocrine disorders and were weight stable for at least the preceding 3 months. Highly restrained eaters, identified by the Dutch Eating Behaviour Questionnaire ${ }^{(10)}$, were excluded from the study. The participants included in the study had a mean score of $2.1($ SEM 0.2$)$ on the restraint scale, 1.98 (SEM $0 \cdot 1$ ) on the emotional scale and $3 \cdot 1$ (SEM 0.1) on the external eating scale. The present study was conducted according to the guidelines laid down in the Declaration of Helsinki and all procedures involving human subjects were approved by the University of Surrey's Ethics Committee; written, informed consent was obtained from all subjects.

\section{Study design}

Subjects attended the investigation unit after an overnight fast, on two occasions, at least 1 week apart, when they consumed either the RS supplement or the placebo supplement within the test breakfast and lunch meals, which provided $24 \mathrm{~g} \mathrm{RS}$ at breakfast and $24 \mathrm{~g}$ RS at lunch on the RS leg. Blood samples and appetite ratings, including desire for different foods, (measured using $100 \mathrm{~mm}$ visual analogue scales, as described by Flint et al. ${ }^{(11)}$ ), were taken every $30 \mathrm{~min}$ for the 7 -h intervention period. The participants remained in the investigation unit for the duration of the study and were required to minimise activity.

On the day before each study day, each participant consumed an identical evening meal and was required to avoid alcohol, caffeine and strenuous exercise for at least $24 \mathrm{~h}$ before the study.

Table 1. Subject measurements taken on the morning of the first study visit

(Mean values with their standard errors for twenty male subjects)

\begin{tabular}{lrl}
\hline Measurement & Mean & SEM \\
\hline Age (years) & 25.8 & 0.82 \\
Height $(\mathrm{cm})$ & 181.2 & 1.62 \\
Weight $(\mathrm{kg})$ & 76.2 & 2.48 \\
BMI $\left(\mathrm{kg} / \mathrm{m}^{2}\right)$ & 23.2 & 0.65 \\
Waist circumference $(\mathrm{cm})$ & 85.5 & 2.07 \\
Hip circumference $(\mathrm{cm})$ & 97.4 & 1.42 \\
Body fat $(\%)^{*}$ & 15.0 & 1.17 \\
Systolic blood pressure $(\mathrm{mmHg}) \dagger$ & 120.8 & 1.69 \\
Diastolic blood pressure $(\mathrm{mmHg}) \dagger$ & 74.4 & 2.16 \\
\end{tabular}

* Measured by bioimpedance (Tanita TBF-300, Tanita, UK).

† Mean of three readings taken with the subject in a sitting position, measured by an automatic blood pressure cuff (Omron MX3 Plus; Omron Healthcare Europe, Kruisweg, UK).
On the two separate days, subjects received either $80 \mathrm{~g}$ Hi-Maize ${ }^{\circledR} 260$ product (60\% RS type 2 and $40 \%$ rapidly digestible starch (RDS)) which therefore provided $48 \mathrm{~g}$ RS and $32 \mathrm{~g}$ RDS (as measured by The Association of Official Analytical Chemists for total dietary fiber method 991.43) or $32 \mathrm{~g}$ of the placebo Amioca ${ }^{\circledR}(100 \%$ RDS) providing $32 \mathrm{~g}$ RDS. Both the supplements were supplied by the National Starch Company, LLC (Bridgewater, NJ, USA) and were incorporated into a flavoured mousse. Available carbohydrate from the RDS portion of both supplements was exactly matched on both occasions, giving meals with comparable glycaemic load. The mousses were of a similar taste and texture and were consumed as part of the test breakfast and lunch meals. Forty grams of the Hi-Maize supplement were the highest quantity that could be added to one mousse portion without adverse effects on taste or texture and a similar high level of RS has been given in other studies without adverse gastrointestinal effects. Subjects were able to choose their preferred flavour of mousse from three choices to enhance compliance, but consumed the same flavour on both visits.

Subjects were cannulated; two fasting blood samples were taken, one $15 \mathrm{~min}$ before breakfast and the other just before breakfast, which was served at time zero. The breakfast on both days was of a standardised portion size and consisted of Rice Krispies ${ }^{\circledR}$ (Kellogg's, Manchester, UK) with semiskimmed milk and one portion of the mousse which contained the test carbohydrate; the energy and macronutrient composition of this meal is shown in Table 2.

Lunch, containing the second half of the test carbohydrate, was served at $180 \mathrm{~min}$, to allow the participants to receive their meals at times similar to their habitual pattern. The lunch meal was either ham or cheese sandwiches (the same filling was consumed on each study day by the same subject), crisps, an orange flavoured drink and one portion of the mousse with the supplement. All food was weighed before being given to the subjects and on the first visit subjects were able to regulate their intake from the offered food (except for the mousse which they were required to fully consume on all visits). Whatever was not consumed was weighed, and the subjects were then required to consume an identical amount on the subsequent visit to ensure the energy and macronutrient intake was identical with only the presence of the RS differing. A similar study design has been used successfully in previous studies ${ }^{(12)}$. The mean values for the amounts consumed at lunch are shown in Table 2 .

At the end of the intervention $(420 \mathrm{~min})$ participants were placed in individual areas and provided with a large preweighed ad libitum homogenous test meal, in excess of normal portion sizes (the whole dish provided: $9765 \mathrm{~kJ}$ energy, $81.5 \mathrm{~g}$ protein; $339.1 \mathrm{~g}$ carbohydrate; $70.0 \mathrm{~g}$ fat; $15.9 \mathrm{~g}$ fibre). Subjects were instructed to consume freely until comfortably full and then the leftovers were weighed. The subjects were informed that they could take home any leftover test meal in order to prevent over-consumption. The ad libitum test meal was a pasta-based meal which was made to a standard recipe with standard cooking times; however, while the energy content was identical on both visits, the energy density per gram would have varied depending on the amount of water absorbed during cooking. Therefore, this was taken into consideration when the energy intake was calculated for each visit. 
Table 2. Nutritional composition of the breakfast and lunch meals consumed on both study days

(Mean values with their standard errors for twenty subjects)

\begin{tabular}{lccccc}
\hline & \multicolumn{2}{c}{ Breakfast } & & \multicolumn{2}{c}{ Lunch } \\
\cline { 2 - 3 } \cline { 6 - 7 } \cline { 6 - 7 } & Mean & SEM & & Mean & SEM \\
\hline Energy (kJ) & 1595 & 2.7 & & 3865 & 151.9 \\
Protein (g) & 9.6 & 0.03 & & 35.6 & 1.54 \\
Carbohydrate $(\mathrm{g})$ & 67.2 & 0.20 & & 111.9 & 3.36 \\
Fat $(\mathrm{g})$ & 7.9 & 0.03 & & 36.8 & 2.87 \\
Fibre $(\mathrm{g})$ & $0.5\left(24.5^{\star}\right)$ & 0.05 & & $5.0\left(29.0^{\star}\right)$ & 0.20 \\
\hline
\end{tabular}

* Resistant starch meal only.

Overall 24-h intake on the study days was assessed from weighed intakes from the breakfast, lunch and ad libitum dinner provided and from diet diaries completed during the late evening by subjects after they had left the unit. All dietary analysis was performed using WinDiets Professional Version program (Robert Gordon University, Aberdeen, UK). Bowel habit diaries were completed on the day of the study and the following day for assessment of gastrointestinal tolerance.

\section{Biochemistry}

Whole blood for glucose analysis was collected into sodium oxalate tubes, for insulin analysis into potassium EDTA tubes and for C-peptide analysis into potassium EDTA tubes with 200 kallikrein inhibiting units (KIU) aprotinin per $\mathrm{ml}$ of whole blood (Trasylol; Bayer, Newbury, UK). All samples were centrifuged for $10 \mathrm{~min}$ at $3000 \mathrm{rpm}$ and then plasma aliquots were stored at $-20^{\circ} \mathrm{C}$ until batch analysis to reduce inter-assay variation. Plasma glucose was measured enzymatically using a commercially available kit (Instrumentation Laboratory, Warrington, UK) for the ILab650 (Instrumentation Laboratory) and the inter-assay variation was $<2 \%$. Concentrations of plasma insulin and C-peptide were measured by RIA with commercially available kits (Millipore; Watford, UK). The sensitivity of the insulin assay was $12 \mathrm{pmol} / \mathrm{l}$ $(2 \mu \mathrm{U} / \mathrm{ml})$ and for the C-peptide assay was $0 \cdot 1 \mathrm{ng} / \mathrm{ml}$, with an inter- and intra-assay variation of $<10 \%$.

\section{Calculations and statistical analysis}

Fasted insulin sensitivity and $\beta$-cell function (homeostasis model assessment (HOMA) \% S and HOMA \% B, respectively) were assessed by $\mathrm{HOMA}^{(13)}$ at the beginning of each study morning; while postprandial insulin sensitivity was assessed using the minimal model method described by Caumo et al. ${ }^{(14)}$.

Area under the curve was calculated for glucose, insulin and $\mathrm{C}$-peptide, using the trapezoid method. The ratio of $\mathrm{C}$-peptide to insulin was calculated using the area under the curve for $2 \mathrm{~h}$ after each meal for both measures and used as a marker of hepatic insulin clearance. Time course data were analysed by repeated measures ANOVA. The data were normally distributed and paired $t$ tests were used to compare between the groups. Statistical analyses were carried out using SPSS version 12.0.1 for Windows (SPSS, Inc., Chicago, IL, USA), with significance assumed as $P<0 \cdot 05$. All the results are means with their standard errors.

\section{Results}

Both the supplements were well tolerated by the subjects with no adverse gastrointestinal effects reported on the day of the study or the following day.

The fasting insulin sensitivity and $\beta$-cell function (assessed by the homeostatic model assessment ${ }^{(13)}$ ) were not significantly different at the start of each of the study days (Table 3), which confirms that the subjects were in a similar metabolic state at the start of each study day.

\section{Energy and macronutrient intake}

Supplementation with $48 \mathrm{~g}$ RS over two meals resulted in a significantly lower energy intake, 5241 (SEM $313 \mathrm{~kJ}$ ) at the offered ad libitum test meal compared to the energy intake seen with the placebo, 5606 (SEM $345 \mathrm{~kJ} ; P=0 \cdot 033$ ).

Over the whole 24 -h period there was also a significantly lower energy intake following the $48 \mathrm{~g}$ RS supplement compared to the placebo supplement, from 12603 to $13949 \mathrm{~kJ}(P=0.044$; Table 4$)$. With the RS leg the mean energy intake was $104 \%$ of calculated habitual energy requirements (calculated with the Schofield equation ${ }^{(15)}$ and a moderate activity level of 1.6 for all subjects) compared to $116 \%$ for the placebo leg.

The lower 24-h energy intake appeared to be mainly due to a significantly lower fat intake, $13 \cdot 3 \mathrm{~g}$ lower, following the RS supplement compared to the placebo (Table 4). The dietary fibre intake during this $24-\mathrm{h}$ period was significantly different

Table 3. Indices of insulin sensitivity following consumption of $48 \mathrm{~g}$ resistant starch (RS) or placebo* (Mean values with their standard errors in twenty healthy, young adult males)

\begin{tabular}{|c|c|c|c|c|c|}
\hline & \multicolumn{2}{|c|}{$\mathrm{RS}$} & \multicolumn{2}{|c|}{ Placebo } & \multirow[b]{2}{*}{$P$} \\
\hline & Mean & SEM & Mean & SEM & \\
\hline HOMA \% S & 95.9 & $7 \cdot 5$ & $97 \cdot 0$ & 8.9 & NS \\
\hline HOMA \% B & $105 \cdot 3$ & 5.9 & 111.4 & $9 \cdot 8$ & NS \\
\hline C-peptide:insulin AUC 0-300 min & $6 \cdot 69$ & 0.37 & $6 \cdot 13$ & 0.42 & 0.059 \\
\hline Oral $S_{1}$ breakfast (dl/kg min per $\left.\mu U \mathrm{ml}\right)$ & $3.36 \times 10^{-3}$ & $0.44 \times 10^{-3}$ & $8.50 \times 10^{-3}$ & $5.58 \times 10^{-3}$ & NS \\
\hline Oral $S_{l}$ lunch $(\mathrm{dl} / \mathrm{kg}$ min per $\mu \cup \mathrm{ml})$ & $5.65 \times 10^{-3}$ & $1.11 \times 10^{-3}$ & $4.43 \times 10^{-3}$ & $0.63 \times 10^{-3}$ & NS \\
\hline
\end{tabular}

HOMA \% S, fasted oral insulin sensitivity, assessed by homeostasis model assessment; HOMA \% B, $\beta$-cell function, assessed by homeostasis model assessment; AUC, area under the curve; $S_{1}$, oral insulin sensitivity to each meal, calculated with a minimal model.

${ }^{*}$ Comparisons were made with a paired samples $t$ test. 
Table 4. Total $24-\mathrm{h}$ intake following supplementation with $48 \mathrm{~g}$ resistant starch (RS) or placebo, measured from 24-h diet diaries*

(Mean values with their standard errors for twenty subjects)

\begin{tabular}{|c|c|c|c|c|c|}
\hline & \multicolumn{2}{|c|}{ RS } & \multicolumn{2}{|c|}{ Placebo } & \multirow[b]{2}{*}{$P$} \\
\hline & Mean & SEM & Mean & SEM & \\
\hline Energy (kJ) & 12603 & 519 & 13949 & 755 & 0.044 \\
\hline Protein (g) & 104.9 & 5.63 & $115 \cdot 3$ & 6.87 & NS \\
\hline Carbohydrate (g) & 424.4 & 18.40 & $452 \cdot 6$ & 23.00 & NS \\
\hline Fat $(g)$ & 96.7 & 4.56 & $110 \cdot 0$ & 4.79 & 0.017 \\
\hline Saturated fat (g) & $39 \cdot 2$ & $2 \cdot 18$ & $45 \cdot 2$ & $2 \cdot 37$ & 0.014 \\
\hline Dietary fibre $(\mathrm{g})$ & $65 \cdot 1$ & 0.95 & $16 \cdot 7$ & 0.84 & $<0.001$ \\
\hline
\end{tabular}

${ }^{*}$ Comparisons were made with a paired samples $t$ test.

due to the supplementation with $48 \mathrm{~g} \mathrm{RS}$, and there did not appear to be a difference in fibre intake by the subjects once they left the investigation unit at the end of the postprandial study period.

\section{Subjective appetite measures}

There was no difference in the subjective appetite scores, measured by the visual analogue scales, for hunger (Fig. 1), fullness, prospective food consumption, thirst or desire for different foods (sweet, salty, savoury or fatty foods) between the two supplements.

\section{Postprandial metabolites}

The postprandial glucose concentrations were not significantly different between the RS supplement and the placebo supplement (Fig. 2(a)).

There was a significantly lower postprandial insulin response following the RS supplementation compared to the placebo supplement over the whole acute study period $(P=0.029$; Fig. 2(b)). However, the corresponding C-peptide concentrations were not significantly different between the two supplements. Consequently, there was an increase in molar ratio of C-peptide to insulin as a surrogate marker for hepatic insulin clearance (Table 3); however, there was no significant difference in postprandial oral insulin sensitivity between the two supplements at either meal (Table 3).

\section{Discussion}

The present study found that after consumption of $48 \mathrm{~g}$ RS (split equally over the test breakfast and lunch meals), there was a lower energy intake at both the ad libitum test meal and over the whole 24-h period, without an associated effect on subjective appetite ratings; the study also found a significant effect of the RS supplement on lowering the postprandial insulin response. To our knowledge this is the first study where RS has been provided to participants as part of a mixed meal and compared to a placebo, where available carbohydrate and energy load have been matched.

Previous studies investigating the effects of RS on appetite have replaced proportions of carbohydrate with RS and therefore the amount of glycaemic carbohydrate provided has varied between the supplements which would confound the interpretation of the results. Indeed, in one study consumption of RS appeared to cause a reduction in subjective feelings of satiety $^{(16)}$; however, a limitation of the study was that the RS and the corresponding $100 \%$ digestible starch were mixed into fruit syrup drinks as a means of delivering the starches, which resulted in the two supplement drinks having a different texture, one liquid and the other semi-solid, with liquids being known to be less satiating than solid foods ${ }^{(17)}$; this study also matched the supplements by weight of starch and therefore the supplements differed in energy and available carbohydrate content. In another study by de Roos et al. ${ }^{(18)}$ two types of RS (RS type 2 and RS type 3) were compared to glucose and, while the supplements were matched for total carbohydrate content, the proportion of available carbohydrate was lower in both of the RS supplements. The study also found that after consumption of each product for 1 week, there was little effect of the RS on appetite. Other studies have investigated the effects on appetite of varying the amylose to amylopectin ratios. One of these studies found that immediately after the meal the high level of amylase was more satisfying than the low amylase but that

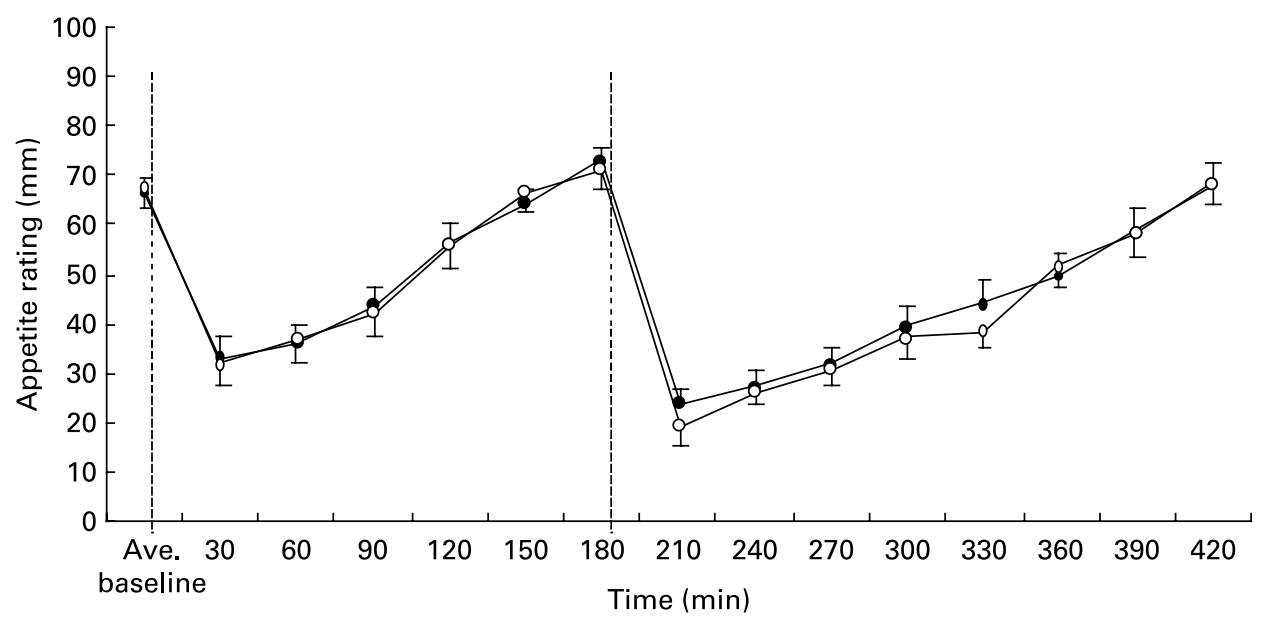

Fig. 1. Subjective appetite ratings on a visual analogue scale in response to the question 'how hungry do you feel?'. Means with their standard errors for twenty healthy, young adult males after consumption of $48 \mathrm{~g}$ resistant starch (RS; $\bullet$ ) compared to a placebo (O). ---, Supplements consumed at the breakfast and lunch meals. There was no significant difference between the supplements for any of the subjective appetite ratings despite the lower energy intake seen with RS consumption. 

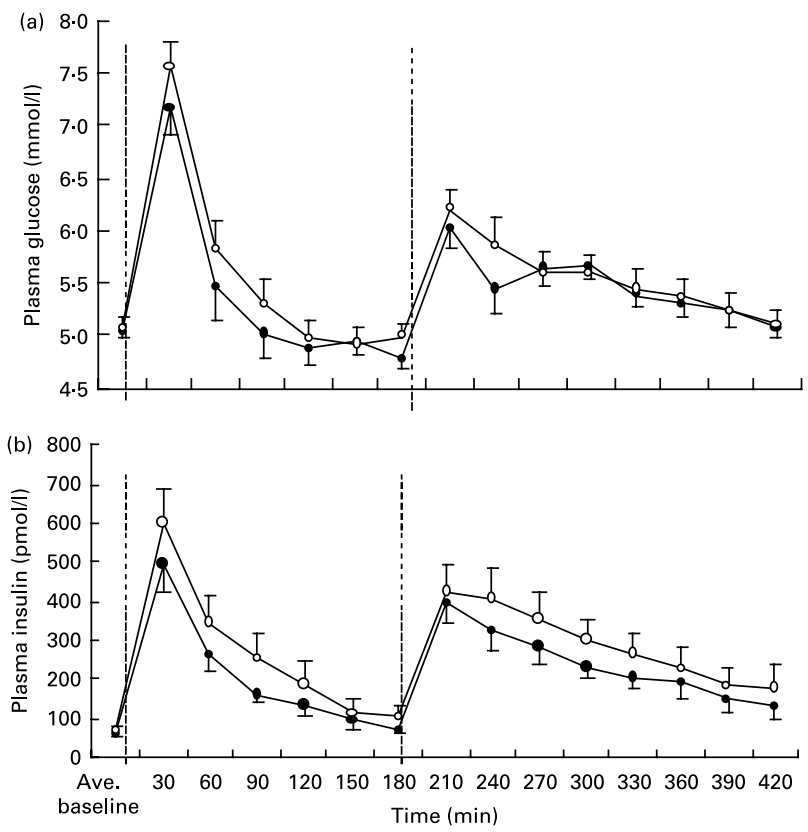

Fig. 2. Postprandial plasma glucose (a) and insulin concentrations (b) after consumption of $48 \mathrm{~g}$ resistant starch (RS; $\bullet$ ) compared to a placebo (O). Means with their standard errors for twenty healthy, young adult males. ---, Supplements consumed at the breakfast and lunch meals. There was no significant difference between the supplements for the glucose concentrations. However, the plasma insulin response was significantly lower $(P=0.029)$ following RS supplementation. Comparisons made with repeated measures ANOVA.

the high amylose was also the least palatable ${ }^{(19)}$. Another study that varied amylose and amylopectin ratios, found no significant effect between their treatments on visual analogue scale ratings ${ }^{(12)}$.

In the present study, on both study days, the participants consumed more than their estimated requirements (104\% on the RS leg and $116 \%$ on the placebo leg) and therefore there was likely to be an element of over-consumption as the participants were given food and did not have to prepare it for themselves; however, the individuals still appeared to over-consume less on the RS leg than on the placebo leg and this can be directly attributed to the RS as the only difference between the test meals. However, this over-consumption may explain why no effects were found in any of the subjective appetite ratings. The overall $24-\mathrm{h}$ intake was derived from the weighed food given to participants at the three meals within the unit and from the reported participant intake later in the evening, so only a small contribution to total intake was obtained indirectly, from participant self-reporting. When using the Goldberg cut-off ${ }^{(20)}$ to identify under-reporting none of the participants would have been classified as under-reporters for either leg of this intervention and so the results are likely to be representative of true intake. All meals were based on standard portion sizes rather than set to BMR, as one of the main outcomes of the study was an effect on the metabolic response, which would have been masked if intakes were set to BMR. The only difference between the two legs, apart from the $48 \mathrm{~g}$ of RS, could have been a small difference in the energy density of the meals due to the presence of the RS; however, this was minor (for breakfast and lunch combined the density per gram was
$7.2 \mathrm{~kJ} / \mathrm{g}$ for the RS leg and $7.7 \mathrm{~kJ} / \mathrm{g}$ for the placebo leg) and the glycaemic load of the two legs was identical.

The lower energy intake seen in the present study over $24 \mathrm{~h}$ appeared to be mainly explained by a significantly lower fat intake following the RS supplement. However, the reason for this lower fat intake is unclear, as the participants did not report a difference in desire for fatty foods on the subjective appetite ratings during the postprandial study. This difference in macronutrient choice following $\mathrm{RS}$ consumption would require further investigation to determine whether it was an incidental finding or a true effect. There also did not appear to be an influence of the high RS intake on fibre intake later in the day; however, there may have been an effect on the following day's fibre intake that was not monitored in the present study.

Although in the present study a reduced energy intake was found following consumption of the RS supplement, it is not possible from the design of the present study to determine exactly what the mechanisms are for the observed effect on food intake. However, as the main effect on food intake appeared to occur at the ad libitum dinner and then later in the day once the participants had left the unit, a possible mechanism could be the fermentation in the colon by colonic microflora and the subsequent effects of SCFA, which has been hypothesised as a mechanism for the effects of fibre on appetite $^{(3)}$.

While the metabolism of SCFA may result in potential additional energy being provided to the body, this was not accounted for in the present study and no value was assigned for the fermentation of the RS. The actual amount delivered to each participant is likely to vary, dependant on the extent of fermentation and metabolism within the colon ${ }^{(21)}$ and variations in individuals' gut transit time ${ }^{(22)}$, as well as a loss to the faeces of a proportion of the ingested RS and the SCFA produced. Published works have stated an energy value of $8 \mathrm{~kJ} / \mathrm{g}$ for Hi-Maize ${ }^{\circledR} 260^{(23)}$ accounting for SCFA metabolism compared to the $16 \mathrm{~kJ} / \mathrm{g}$ for the placebo. In the present study, we matched the supplements by glycaemic load as the largest confounder to the glycaemic response. When taking into consideration the amounts of each supplement given this would have resulted in maximal energy doses of $640 \mathrm{~kJ}$ for the RS and $512 \mathrm{~kJ}$ for the placebo, with a difference of only $128 \mathrm{~kJ}$. This therefore does not account for the full difference in energy intake observed in the study over the $24-\mathrm{h}$ period which was $1346 \mathrm{~kJ}$.

It is possible that the increase in production of SCFA may consequently increase production of anorexigenic hormones from the colon, such as peptide $\mathrm{YY}^{(24,25)}$. However, so far the only evidence of an effect of RS on these hormones, particularly peptide YY and glucagon-like peptide, has been shown in rodent studies ${ }^{(26-28)}$, which are, in terms of the gastrointestinal tract, anatomically different ${ }^{(29)}$ to human subjects. Human studies which have measured gastrointestinal peptides have found no effect of fibre feeding ${ }^{(6,16)}$.

As insulin and C-peptide are co-secreted, the lower postprandial insulin concentration detected was likely to be due to increased hepatic insulin clearance, as there was a trend towards significance between the two supplements for the molar ratio of C-peptide to insulin. An increase in hepatic insulin clearance has previously been reported following RS intake over $24 \mathrm{~h}^{(30)}$. It has been proposed that the increase in 
production of SCFA and their exposure to the liver may ultimately be responsible for the increase in insulin clearance.

The lower energy intake seen in the present study following RS consumption as part of a mixed meal could have beneficial implications in weight management and, potentially, weight loss; however, further studies are required to confirm whether a similar finding is seen in other population groups such as the overweight or obese, and to determine the actual mechanisms for the effect. Although the dose in the present study was well tolerated over the one day of the study and a lower energy intake was observed, further investigations are needed to establish whether the dose has similar effects when ingested chronically. A lower postprandial insulin response was also observed which could be explained by an increase in hepatic insulin clearance. Increased intakes of RS in the diet may therefore have beneficial implications in weight management.

\section{Acknowledgements}

We thank all of the participants for their time. C. L. B. was involved in designing the study protocol, recruitment of volunteers, conducting the study days, analysis of data and writing the paper. G. S. F. was involved in obtaining the funding, the protocol and refining the paper. M. D. R. was involved in designing the study protocol, assistance on several of the study days and analysis of data, and refining the paper. There were no conflicts of interest. C. L. B. was supported by an educational fellowship from Premier Foods and supplements were supplied by National Starch Company, LLC.

\section{References}

1. World Health Organization (WHO) (2000) Nutrition for Health and Development. A Global Agenda for Combating Malnutrition. Geneva: World Health Organization. http://www.who. int/mip2001/files/2231/NHDprogressreport2000.pdf (accessed August 2007).

2. Slavin J \& Green H (2007) Dietary fibre and satiety. Nutr Bull 32, 32-42.

3. Pereira MA \& Ludwig DS (2001) Dietary fiber and body-weight regulation. Observations and mechanisms. Pediatr Clin North Am 48, 969-980.

4. Weickert MO \& Pfeiffer AF (2008) Metabolic effects of dietary fiber consumption and prevention of diabetes. J Nutr 138, 439-442.

5. Champ MM (2004) Physiological aspects of resistant starch and in vivo measurements. J AOAC Int 87, 749-755.

6. Robertson MD, Bickerton AS, Dennis AL, et al. (2005) Insulinsensitizing effects of dietary resistant starch and effects on skeletal muscle and adipose tissue metabolism. Am J Clin Nutr 82, 559-567.

7. Brown IL (2004) Applications and uses of resistant starch. J AOAC Int 87, 727-732.

8. Nugent AP (2005) Health properties of resistant starch. Nutr Bull 30, 27-54.

9. Higgins JA (2004) Resistant starch: metabolic effects and potential health benefits. J AOAC Int 87, 761-768.

10. van Strien T, Frijters JER, Bergers GPA, et al. (1986) The Dutch Eating Behavior Questionnaire (DEBQ) for assessment of restrained, emotional, and external eating behavior. Int $J$ Eat Disord 52, 295-315.
11. Flint A, Raben A, Blundell JE, et al. (2000) Reproducibility, power and validity of visual analogue scales in assessment of appetite sensations in single test meal studies. Int $J$ Obes Relat Metab Disord 24, 38-48.

12. Weststrate JA \& van Amelsvoort JM (1993) Effects of the amylose content of breakfast and lunch on postprandial variables in male volunteers. Am J Clin Nutr 58, 180-186.

13. Matthews DR, Hosker JP, Rudenski AS, et al. (1985) Homeostasis model assessment: insulin resistance and beta-cell function from fasting plasma glucose and insulin concentrations in man. Diabetologia 28, 412-419.

14. Caumo A, Bergman RN \& Cobelli C (2000) Insulin sensitivity from meal tolerance tests in normal subjects: a minimal model index. J Clin Endocrinol Metab 85, 4396-4402.

15. Schofield WN, Schofield C \& James WPT (1985) Basal metabolic rate - review and predicition. Hum Nutr: Clin Nutr 39, $1-96$.

16. Raben A, Tagliabue A, Christensen NJ, et al. (1994) Resistant starch: the effect on postprandial glycemia, hormonal response, and satiety. Am J Clin Nutr 60, 544-551.

17. Mourao DM, Bressan J, Campbell WW, et al. (2007) Effects of food form on appetite and energy intake in lean and obese young adults. Int J Obes (Lond).

18. De Roos N, Heijnen ML, de Graaf C, et al. (1995) Resistant starch has little effect on appetite, food intake and insulin secretion of healthy young men. Eur J Clin Nutr 49, $532-541$.

19. van Amelsvoort JM \& Weststrate JA (1992) Amylose-amylopectin ratio in a meal affects postprandial variables in male volunteers. Am J Clin Nutr 55, 712-718.

20. Goldberg GR, Black AE, Jebb SA, et al. (1991) Critical evaluation of energy intake data using fundamental principles of energy physiology: 1. Derivation of cut-off limits to identify under-recording. Eur J Clin Nutr 45, 569-581.

21. Wolever TM, Schrade KB, Vogt JA, et al. (2002) Do colonic short-chain fatty acids contribute to the long-term adaptation of blood lipids in subjects with type 2 diabetes consuming a high-fiber diet? Am J Clin Nutr 75, 1023-1030.

22. Macfarlane S \& Macfarlane GT (2003) Regulation of shortchain fatty acid production. Proc Nutr Soc 62, 67-72.

23. Behall KM \& Howe JC (1996) Resistant starch as energy. $J$ Am Coll Nutr 15, 248-254.

24. Cuche G, Cuber JC \& Malbert CH (2000) Ileal short-chain fatty acids inhibit gastric motility by a humoral pathway. Am $J$ Physiol Gastrointest Liver Physiol 279, G925-G930.

25. Cherbut C (2003) Motor effects of short-chain fatty acids and lactate in the gastrointestinal tract. Proc Nutr Soc 62, 95-99.

26. Keenan MJ, Zhou J, McCutcheon KL, et al. (2006) Effects of resistant starch, a non-digestible fermentable fiber, on reducing body fat. Obesity 14, 1523-1534.

27. Zhou J, Hegsted M, McCutcheon KL, et al. (2006) Peptide YY and proglucagon mRNA expression patterns and regulation in the gut. Obesity (Silver Spring) 14, 683-689.

28. Zhou J, Martin RJ, Tulley RT, et al. (2008) Dietary resistant starch upregulates total GLP-1 and PYY in a sustained daylong manner through fermentation in rodents. Am J Physiol Endocrinol Metab 295, E1160-E1166.

29. Topping DL \& Clifton PM (2001) Short-chain fatty acids and human colonic function: roles of resistant starch and nonstarch polysaccharides. Physiol Rev 81, 1031-1064.

30. Robertson MD, Currie JM, Morgan LM, et al. (2003) Prior short-term consumption of resistant starch enhances postprandial insulin sensitivity in healthy subjects. Diabetologia 46, 659-665. 